\title{
Tender puentes: lecciones globales desde México sobre políticas de salud basadas en evidencias
}

\author{
Julio Frenk, MC, PhD. (I)
}

\begin{abstract}
Frenk J.
Tender puentes: lecciones globales desde México sobre políticas de salud basadas en evidencias. Salud Publica Mex 2007;49 supl I:S I4-S22.
\end{abstract}

\section{Resumen}

Durante los últimos seis años, México ha llevado a cabo una profunda transformación de su sistema de salud. Este artículo presenta una visión general de las principales características de la experiencia de reforma mexicana. Como resultado del alto grado de desigualdad social, México es un microcosmos de la diversidad de problemas que afectan a los países de todos los niveles de desarrollo. Su sistema de salud no estaba a la altura de las presiones impuestas por la doble carga de enfermedad, en donde la desnutrición, las infecciones comunes y los problemas de salud reproductiva coexisten con las enfermedades no transmisibles y las lesiones. Con la mitad de su población sin acceso al aseguramiento en salud, México enfrentaba una paradoja inaceptable: si bien la salud es un factor clave en la lucha contra la pobreza, un gran número de familias se empobrecía como consecuencia de los gastos para la atención de su salud, incluyendo los medicamentos. La reforma se diseñó para corregir esta paradoja mediante la introducción de un innovador esquema denominado Seguro Popular, a través del cual se protege de manera gradual a 50 millones de mexicanos, la mayoría de ellos pobres, que hasta ahora habían sido excluidos de la seguridad social formal. Este artículo presenta una serie de resultados alentadores para lograr el fin último de la reforma: el acceso universal a servicios de salud de alta calidad con protección social para todos.

Palabras clave: reforma del sistema de salud; programas nacionales de salud; acceso universal a servicios de salud; políticas de salud; México
Frenk J.

Bridging the divide: global lessons

from evidence-based health policy in Mexico.

Salud Publica Mex 2007;49 suppl I:SI 4-S22.

\section{Abstract}

During the past six years, Mexico has undergone a largescale transformation of its health system. This paper provides an overview of the main features of the Mexican reform experience. Because of its high degree of social inequality, Mexico is a microcosm of the range of problems that affect countries at all levels of development. Its health system had not kept up with the pressures of the double burden of disease, whereby malnutrition, common infections, and reproductive health problems coexist with non-communicable disease and injury. With half of its population uninsured, Mexico was facing an unacceptable paradox: whereas health is a key factor in the fight against poverty, a large number of families became impoverished by expenditures in health care and drugs. The reform was designed to correct this paradox by introducing a new scheme called Popular Health Insurance (Seguro Popular). This innovative initiative is gradually protecting the 50 million Mexicans, most of them poor, who had until now been excluded from formal social insurance. This paper reports encouraging results in the achievement of the ultimate objective of the reform: universal access to high-quality services with social protection for all.

Key words: health system reform; national health programs; universal access to health care services; health policy; Mexico 
$\mathrm{H}^{2}$ oy más que nunca, el mundo necesita respuestas integrales a los complejos problemas que enfrenta. Pocos campos ilustran esta necesidad en forma tan clara como los esfuerzos nacionales y mundiales por mejorar la salud. Han transcurrido ya tres quintas partes del plazo para cumplir con los Objetivos de Desarrollo del Milenio. A estas alturas, la mayoría de los países siguen luchando para mantener un curso de acción que permita alcanzar los resultados deseados. ${ }^{1}$ Sin embargo, se trata de plazos y resultados que nadie puede darse el lujo de no cumplir.

En particular, los ministros de salud de todo el mundo enfrentan desafíos sin precedentes en la medida que buscan convertirse en rectores efectivos de sus sistemas nacionales de salud. Los perfiles de enfermedad se vuelven cada vez más diversos y complejos, y las presiones crecen para desarrollar sistemas de salud que respondan con equidad, calidad y protección financiera a las necesidades y expectativas de la población.

Durante casi seis años, México ha estado inmerso en un proceso de transformación de su sistema de salud que puede contener lecciones importantes para otras naciones en vías de desarrollo. México es un país heterogéneo, de ingreso medio, con una población de más de 100 millones. Su alto grado de desigualdad social significa que en él se refleja toda la gama de problemas de salud que afectan al mundo. Como la mayoría de los países en vías de desarrollo, México enfrenta una transición epidemiológica prolongada y desigual ${ }^{2}$ que está sumando nuevas capas de complejidad a los patrones de enfermedad, discapacidad y muerte. A través de una red de multicausalidad, estos países deben encarar una doble carga de enfermedad: por un lado, la agenda inconclusa de infecciones, desnutrición y problemas de salud reproductiva; por el otro, los retos emergentes representados por las enfermedades no transmisibles (asociados con factores de riesgo como el tabaquismo y la obesidad), los trastornos mentales y el flagelo creciente de las lesiones y la violencia. ${ }^{3}$

La complejidad en las condiciones de salud tiene su paralelo en la complejidad de la organización, financiamiento y gestión de los sistemas de salud. Sabemos que el desempeño de los sistemas de salud varía mucho de un país a otro, incluso entre aquéllos con el mismo nivel de ingreso y gasto en salud. ${ }^{4}$ Dependiendo de este desempeño, una sociedad puede enfrentar un ciclo ya sea virtuoso o vicioso entre su nivel de desarrollo y la forma como funciona su sistema de salud. La conclusión es clara: las políticas de salud son opciones sociales y la forma en que se formulan e implantan influye en cuál de esos ciclos se genera.
La única manera de encarar estas complejidades crecientes es transcendiendo las diversas dicotomías que se han constituido en obstáculos para el progreso, incluyendo la división entre los enfoques vertical y horizontal, las políticas sectoriales e intersectoriales, el análisis y la acción, y los esfuerzos nacionales y las iniciativas globales. En este artículo, analizo la forma en que una reforma integral se ha encaminado de manera explícita a tender un puente entre las dos caras de estos falsos dilemas.

En el diseño, implantación y evaluación de su reforma, México ha hecho un uso intensivo de las mejores evidencias disponibles, que se ha derivado de análisis nacionales y de diversos bienes públicos globales asociados con el conocimiento, ${ }^{5}$ como las comparaciones sistemáticas de las experiencias de otros países, marcos conceptuales y métodos de medición. En particular, México ha asimilado las lecciones de las innovaciones introducidas en muchos otros países del mundo; al mismo tiempo, ha puesto a disposición de otras naciones sus propias experiencias. Es evidente que los países no pueden simplemente adoptar las experiencias internacionales a sus propias realidades sociales, financieras y culturales; lo que deben hacer es adaptarlas. Aun así, existe una búsqueda mundial por mejores formas de abordar los complejos retos de nuestros tiempos. Debido a las lagunas en nuestro conocimiento, cada iniciativa de reforma debe verse como un experimento cuyos efectos deben documentarse para beneficio de todas las demás iniciativas, tanto presentes como futuras. Este esfuerzo requiere de una sólida inversión en investigación de sistemas de salud. ${ }^{6}$ Cada innovación constituye una oportunidad de aprendizaje; no aprovecharla nos condena a redescubrir, a un enorme costo, lo que ya se sabía o a repetir los errores del pasado. Para reformar, es indispensable informar, de lo contrario se corre el peligro de deformar. Al poner nuestras experiencias al alcance de la comunidad internacional, esperamos contribuir a un proceso de aprendizaje compartido entre los países que será esencial para alcanzar nuestras metas comunes en salud.

\section{Nuevas dinámicas de pobreza y salud}

La necesidad de la reforma mexicana surgió de las crecientes presiones impuestas al sistema de salud por la doble carga de enfermedad. Este proceso dinámico se ha vuelto común en la mayoría de los países en vías de desarrollo. En salud, somos víctimas de nuestros propios éxitos. El mejoramiento de las condiciones básicas de salud echa a andar la transición epidemiológica 
al incrementar la sobrevida infantil que permite alcanzar edades en las que las enfermedades no transmisibles son más prevalentes. Dado que la velocidad de este cambio está distribuida en forma desigual entre los diferentes grupos sociales, la población acaba por enfrentar una doble carga de enfermedad. Una realidad que con frecuencia se pasa por alto en la búsqueda de la equidad es que los problemas que sólo afectan a los pobres, como muchas infecciones comunes y la desnutrición, ya no son los únicos problemas de los pobres, quienes también muestran las tasas más elevadas de muchos padecimientos no transmisibles, trastornos mentales, lesiones, violencia, tabaquismo, obesidad y otros factores de riesgo.

En México, como en otros países semejantes, el sistema de salud no estaba a la altura de las crecientes presiones financieras impuestas por esta doble carga. Aunque la seguridad social se introdujo en 1943, ésta se había limitado a los trabajadores asalariados de empresas privadas o de instituciones del sector público y sus familias. Esta configuración excluía a los trabajadores por cuenta propia, los desempleados y a aquellos que estaban fuera del mercado laboral o que trabajaban en el sector informal de la economía. El resultado es que para el año 2000, la mitad de las familias mexicanas, la mayor parte pobre, no contaba con protección social contra las consecuencias financieras de la enfermedad.

Realidades similares a lo largo y ancho del mundo en vías de desarrollo están planteando una paradoja inaceptable: si bien el mejoramiento de la salud es una de las formas más efectivas de superar la pobreza, ${ }^{7}$ la atención médica en sí misma puede convertirse en un factor de empobrecimiento para las familias cuando un país no cuenta con los mecanismos sociales para asegurar un financiamiento justo de la atención a la salud que proteja a toda la población.

\section{Empoderamiento a través de las evidencias}

La reforma del sistema mexicano de salud se diseñó para corregir esta paradoja. Lo hizo a través de una fuerte inversión en la generación y aplicación de conocimiento pertinente, lo que constituye probablemente un caso de "libro de texto" sobre las políticas públicas basadas en evidencias. ${ }^{8}$ En efecto, la combinación de diversos métodos de medición adoptados internacionalmente con análisis nacionales reveló realidades críticas que requerían de solución. Así, el cálculo de las cuentas nacionales en salud mostró que más de la mitad del gasto total en México era gasto de bolsillo. ${ }^{9}$ Éste era resultado directo, como se señaló arriba, del he- cho de que cerca de la mitad de la población carecía de un seguro de salud. ${ }^{10}$ Aún más, los mismos estudios demostraron que estos gastos de bolsillo eran regresivos, ya que representaban una proporción mayor de los ingresos entre los hogares pobres que entre los más ricos. ${ }^{11}$

Estos hallazgos resultaron inesperados, ya que había la creencia generalizada de que el sistema mexicano de salud se financiaba principalmente con fondos públicos. En lugar de eso, el análisis de 2000 mostró que cada trimestre casi 1.5 millones de hogares enfrentaban una catástrofe económica, caían por debajo de la línea de pobreza o se hundían más en la pobreza como consecuencia de pagar por su salud con gastos de bolsillo. ${ }^{12,13}$ De esta manera, dichas evidencias sólidas despertaron la conciencia sobre una realidad que hasta entonces había estado fuera de la agenda pública, a saber: que la misma atención a la salud podía convertirse en causa directa de empobrecimiento. Otros estudios han mostrado una situación similar en la mayoría de los países en vías de desarrollo, en los que cada año cientos de millones de personas caen en una trampa de pobreza porque no cuentan con protección social contra las consecuencias financieras de la enfermedad. ${ }^{14,15}$

En el caso de México, este descubrimiento cambió la agenda al generar una perspectiva diferente sobre la operación del sistema de salud. Los tomadores de decisiones empezaron a mostrar interés por temas financieros que demostraron tener un enorme impacto sobre la atención a la salud y la pobreza en los hogares mexicanos.

Otro bien público global que contribuyó a sustentar la reforma local fue el marco conceptual de la Organización Mundial de la Salud (OMS) para la evaluación del desempeño de los sistemas de salud. ${ }^{16}$ Este marco destaca la justicia en el financiamiento como una de las metas intrínsecas de los sistemas de salud. Como resultado de su alto grado de gasto de bolsillo, México presentó resultados muy pobres en el análisis comparativo internacional sobre justicia financiera. En lugar de generar una reacción defensiva, este resultado estimuló un análisis detallado nacional en 2001, el cual mostró que los gastos catastróficos se concentraban en hogares pobres y no asegurados. Dicho análisis se basó en datos de las encuestas nacionales de ingresos y gastos de los hogares en México, otro bien público global. Estas encuestas se realizan en muchos países del mundo y generan datos homogéneos muy valiosos para el análisis comparativo entre países, pero que no se han explotado lo suficiente para la formulación de políticas públicas de salud. 


\section{De las evidencias a la acción}

La interrelación estrecha entre los análisis nacionales e internacionales generó los instrumentos de promoción de una profunda reforma legislativa para establecer un sistema de protección social en salud, la cual fue aprobada por una amplia mayoría de todos los partidos políticos en el Congreso mexicano. A partir de su entrada en vigor el 1 de enero de 2004, el nuevo sistema se extenderá gradualmente para proteger a $12 \mathrm{mi}-$ llones de familias no aseguradas (cerca de 50 millones de personas) a lo largo de siete años, con lo que se alcanzará la cobertura universal para 2010. El vehículo para lograr este objetivo es un seguro público voluntario denominado Seguro Popular de Salud. Si bien el periodo de transición puede parecer largo, exige enormes esfuerzos organizacionales para afiliar a $1.7 \mathrm{mi}-$ llones de familias por año. Actualmente, en su tercer año de implantación, el Seguro Popular ha despertado una respuesta entusiasta de la población, de manera que para finales de 2006 habrá alcanzado su meta de afiliar a 5.1 millones de familias (alrededor de $22 \mathrm{mi}$ llones de personas). ${ }^{17}$

Para asegurar que esta expansión de la cobertura cuente con los recursos necesarios, la reforma introduce un nuevo esquema financiero basado en el costeo explícito de beneficios. De esta manera, la ley estipula ahora una obligación presupuestaria para satisfacer la demanda esperada de cada familia afiliada. El resultado neto es que el financiamiento público aumentará en 1 punto porcentual completo del producto interno bruto (PIB) en el curso de siete años, en su mayor parte procedente de impuestos federales generales complementados por contribuciones estatales. Este incremento de recursos es posible por el hecho de que el punto de partida para el gasto total en salud era de sólo $5.6 \%$ del PIB en 2000, nivel que resultaba insuficiente para enfrentar las presiones impuestas por la doble carga de enfermedad.

Los beneficios financieros de la reforma ya se están materializando. Entre 2001 y 2003, el gasto público en salud per cápita para la población no asegurada creció a una tasa promedio de sólo $5.2 \%$ por año en términos reales (insuficiente para mantener el paso de las transiciones demográfica y epidemiológica). En contraste, durante los primeros tres años de la reforma (2004-2006), la tasa promedio de crecimiento real se ha más que duplicado, al pasar a $12.3 \%$ anual. De acuerdo con las nuevas disposiciones legales, se proyecta que dicha tasa se mantendrá en cerca de 10\% anual hasta 2010. Gracias a la reforma, durante la presente administración el presupuesto de la Secretaría de Salud se ha incrementado en más del doble, lo que se traduce en un incremento real, una vez descontada la inflación, de $69 \%{ }^{18}$

La movilización de recursos adicionales encabeza un gran esfuerzo por realinear los incentivos a lo largo del sistema de salud. La afiliación de familias al Seguro Popular es ahora la base para la asignación recursos federales a los estados, responsables de la prestación de servicios. De esta forma, el viejo modelo de "presupuestación burocrática", que subsidiaba a los proveedores independientemente de su desempeño, está siendo reemplazado por una "presupuestación democrática", en donde el dinero sigue a las personas para asegurar el mejor equilibrio entre calidad y eficiencia.

Para lograr este objetivo, actualmente la reforma financiera en el nivel macro se complementa con una reforma gerencial en el nivel micro, la cual fortalece la capacidad prestadora de servicios a través de intervenciones específicas como la planeación de largo plazo de nuevas instalaciones; la evaluación de tecnologías; el suministro eficiente de medicamentos y su prescripción racional; el desarrollo de recursos humanos apropiados, que incluye la capacitación gerencial; el diseño de sistemas de información orientados a resultados; la acreditación de unidades de atención; la certificación de prestadores; el mejoramiento de la calidad en las dimensiones técnica e interpersonal de la atención, y el análisis comparativo del desempeño tanto de los sistemas estatales como de las instituciones de salud. Estos aspectos del sistema de salud han requerido de un fortalecimiento extraordinario para atender mejor a las poblaciones más pobres que viven en las áreas de mayor marginación. Asimismo, todos estos son componentes críticos del papel rector que los ministerios de salud deben cumplir con una creciente competencia.

\section{Derechos legislados y prioridades}

Un elemento articulador de la reforma financiera y gerencial es un paquete específico de beneficios que se diseñó tomando como criterios orientadores el costo, la efectividad y la aceptabilidad social. Además de servir para la definición de prioridades, el paquete es un medio para empoderar a las personas al generar conciencia sobre sus derechos, y es además un instrumento clave para la rendición de cuentas por parte de los proveedores.

Un antecedente de este modelo fue la innovadora iniciativa instrumentada a mediados de los años noventa para fortalecer las capacidades básicas de las familias en extrema pobreza. Denominado en un principio Progresa y luego Oportunidades, este programa crea incentivos en las familias para invertir en el capital 
humano de sus hijos a través de transferencias en efectivo, que se proporcionan bajo la condición de que las familias cumplan con ciertos compromisos de corresponsabilidad, como enviar a los niños a la escuela en lugar de a trabajar, proporcionarles suplementos nutricionales específicamente formulados y acudir a la clínica para recibir un paquete específico de intervenciones para la promoción de la salud y la prevención de enfermedades. Estas intervenciones incluyen higiene básica, salud reproductiva, vigilancia nutricional y del crecimiento, así como medidas preventivas específicas dirigidas en particular a enfermedades transmisibles, pero cada vez con mayor énfasis en hipertensión arterial, diabetes y lesiones. Desde su instauración, el programa ha tenido un componente de evaluación que ha sido lo suficientemente sólido para atribuir mejoras sustanciales a diversas intervenciones y ha generado, al mismo tiempo, evidencias para ajustar su operación. ${ }^{19,20}$

No obstante que Oportunidades demostraba su valor para la reducción de la pobreza y el mejoramiento de la salud, los beneficiarios experimentaban nuevas cargas de enfermedad, en tanto que crecían sus expectativas de una mejor calidad de la atención. Irónicamente, una proporción significativa de las transferencias monetarias que las familias pobres recibían del programa se empleaban para financiar la atención de aquellos padecimientos que no se contemplaban en el paquete básico inicial de intervenciones, el cual se enfocaba más a los patrones pretransicionales de la carga de enfermedad.

Sobre la base de la exitosa plataforma provista por Oportunidades, la protección social para las familias pobres necesitaba expandirse a través del Seguro Popular. El resultado neto ha sido un aumento notable en los alcances de los beneficios. A partir del paquete básico original de Oportunidades de sólo 13 intervenciones, se pasó, con el Seguro Popular, a cubrir más de 250 intervenciones que se ofrecen en los niveles primario y secundario de atención.

\section{En busca de la diagonal}

El paquete específico de beneficios ofrece un medio para tender un puente entre las diferencias representadas por uno de los falsos dilemas mencionados anteriormente, a saber, entre el modelo vertical, que se enfoca en enfermedades específicas, y el horizontal, encaminado a fortalecer de manera general la estructura y las funciones del sistema de salud. Para superar este falso dilema, necesitamos extender la metáfora geométrica y buscar lo que se ha denominado la diagonal, ${ }^{21}$ esto es, una estrategia en la que se aprovecha un conjunto de intervenciones específicas de alta prioridad para impulsar las mejoras necesarias en el sistema de salud.22

El paquete integral de beneficios del Seguro Popular sirve como herramienta para instrumentar el modelo diagonal mediante el fortalecimiento de la capacidad de todo el sistema de salud a través de la definición explícita de prioridades. Los esfuerzos por reducir la mortalidad materna ejemplifican este punto. Al haber logrado ya una cobertura mayor a $95 \%$ con uno de los esquemas de vacunación más completos del mundo, la siguiente frontera para la equidad era acortar la brecha social en mortalidad materna, que es el Objetivo de Desarrollo del Milenio que requiere del mayor esfuerzo por parte de México. Por consiguiente, se lanzó una iniciativa especial para abordar esta prioridad esencial, enfocando los diversos componentes financieros y gerenciales de la reforma en un conjunto de intervenciones comunitarias y hospitalarias. Las evaluaciones iniciales revelan una aceleración importante en la tasa de descenso de la mortalidad materna, de una reducción promedio de sólo 1.8\% anual entre 1990 y 2000, a 3.9\% para el periodo 20002005. Es claro que este resultado positivo puede atribuirse a otros factores además de la iniciativa especial; no obstante, el resultado neto es que el progreso más rápido durante los últimos cinco años se traduce en una reducción de $20 \%$ en el número absoluto de muertes maternas. ${ }^{23}$

Además de financiar las intervenciones de nivel primario y secundario, los incrementos presupuestales durante el periodo de aumento progresivo de siete años estipulado en la nueva ley permiten extender la cobertura a un conjunto más amplio de intervenciones de alto costo definidas a través de un mecanismo transparente y colectivo de determinación de prioridades. El ejemplo más dramático ha sido el programa contra el VIH/SIDA. Gracias a la participación activa de las organizaciones de la sociedad civil, entre 2000 y 2006 el Congreso mexicano ha aprobado un aumento de 14 veces en el presupuesto dedicado a este programa. Los recursos adicionales se han empleado para sostener una estrategia basada en tres pilares: prevención, campañas contra la discriminación y acceso universal al tratamiento integral, incluidos los antirretrovirales. El tratamiento del SIDA ha sido objeto de una vía de cobertura universal acelerada especial. Gracias a esto, toda persona no asegurada que tenga necesidad de un tratamiento es elegible para afiliarse de inmediato al Seguro Popular. Mientras que en 2000 la Secretaría de Salud proporcionaba terapia antirretroviral solamente a 2386 personas no aseguradas, en la actualidad 15750 
personas lo reciben sin costo alguno, junto con el resto de los beneficios del Seguro Popular.

El mismo esquema de cobertura universal acelerada se aplica al tratamiento del cáncer en niños (que ya es la segunda causa de muerte entre los niños de edad escolar) ${ }_{,}^{24}$ al tratamiento del cáncer cervicouterino (que muestra un descenso de $22 \%$ desde 2000 en la mortalidad entre mujeres mayores de 25 años de edad) ${ }^{25}$ y a la cirugía de cataratas (solución altamente costo-efectiva para la principal causa de ceguera en el adulto), ${ }^{26}$ entre otras intervenciones. Cada intervención mencionada proporciona una oportunidad para mejorar el desempeño general del sistema de salud a través de la consecución de prioridades explícitas.

La cobertura ampliada del Seguro Popular se está reflejando ya en una mayor protección financiera para las familias pobres. Las comparaciones entre diversas rondas de la Encuesta Nacional de Ingresos y Gastos de los Hogares (ENIGH) muestran una reducción de $30 \%$ en el número de hogares del quintil de menores ingresos con gastos catastróficos por la atención a la salud..$^{25}$

\section{Protección de las inversiones en prevención}

Los beneficios del nuevo sistema no se limitan a las acciones curativas. Por primera vez en México el nuevo sistema ha creado un Fondo de Aportaciones para Servicios de Salud a la Comunidad independiente que protege el presupuesto dedicado a las intervenciones de promoción de la salud y prevención de enfermedades. Un riesgo de las reformas financieras es que el incremento en el acceso a los servicios curativos se produzca a costa del presupuesto de las intervenciones para la comunidad, que con frecuencia no generan una demanda espontánea de la población. El fondo independiente se estableció para evitar este tipo de distorsión, que aumenta el costo de la atención curativa al desatender los programas costo-efectivos para la prevención y la detección temprana. Un instrumento complementario es un esquema de cartillas de salud con una perspectiva de género y línea de la vida, que da seguimiento a las medidas preventivas necesarias para cada grupo de edad y género. De hecho, el cumplimiento de estas medidas es un requisito de corresponsabilidad para afiliarse al Seguro Popular.

El énfasis renovado en la prevención produce resultados positivos para los dos componentes de la doble carga de enfermedad. Por el lado de la agenda inconclusa, las prioridades incluyen la tuberculosis (con una reducción de 30\% en la mortalidad durante los últimos cinco años atribuible a las mejoras en la detección de casos y el éxito del tratamiento); paludismo (reducción de $60 \%$ en el número de casos); el cáncer cervicouterino (que muestra un incremento de 32\% en pruebas de Papanicolaou, con la tasa más alta de crecimiento entre las mujeres pobres), y la sobrevivencia infantil. ${ }^{25}$ De los 60 países prioritarios identificados por la Unicef, México destaca como uno de los siete que está en ruta para alcanzar la Meta de Desarrollo del Milenio relacionada con la sobrevivencia infantil. ${ }^{27}$

Por lo que se refiere a los retos emergentes, se pueden evaluar los resultados a través de dos encuestas nacionales de Salud y Nutrición aplicadas en los años 2000 y 2005-2006, las cuales incluyen cuestionarios detallados sobre el uso de los servicios, así como mediciones clínicas y de laboratorio. Durante el periodo entre las dos encuestas (que corresponde a la actual administración de gobierno), se registra un incremento significativo en la utilización de los servicios de detección temprana para varias enfermedades no transmisibles, destacadamente hipertensión ( $52 \%$ de aumento en las mediciones de la presión arterial) y cáncer de mama (un aumento de $71 \%$ en el uso de mastografías). ${ }^{28}$

\section{Políticas saludables}

Las acciones preventivas deben ser parte de una estrategia que tienda otro puente más, en este caso entre las políticas sectoriales y las intersectoriales. En efecto, un componente clave del papel rector que deben asumir los ministros de salud es movilizar todos los instrumentos de política pública y no sólo aquellos bajo su control directo. La salud no puede ser vista simplemente como un sector específico de la administración pública, debe entenderse como un objetivo social. Por consiguiente, desarrollar las políticas de salud en el estricto sentido sectorial no es suficiente; también se necesitan políticas saludables que movilicen intervenciones de otros sectores en busca de una mejor salud.

Por esta razón, la reforma mexicana ha incluido un esfuerzo sin precedente para fortalecer los servicios de salud ambiental, las acciones regulatorias para proteger al público y, en general, el conjunto de intervenciones intersectoriales que define una política saludable capaz de modificar los más amplios determinantes de la enfermedad. Para ello, la Secretaría de Salud ha emprendido una reorganización profunda encaminada al establecimiento de una nueva agencia de salud pública encargada de la protección contra los riesgos a la salud. Esta agencia tiene como objetivos garantizar la inocuidad de los alimentos, definir estándares ambientales y ocupacionales, regular la indus- 
tria farmacéutica y controlar las sustancias peligrosas como el alcohol y el tabaco. El tabaquismo, en particular, ha sido materia de una política integral que incluye un importante incremento de los impuestos a su consumo, la prohibición de publicidad en medios electrónicos, un aumento sustancial en el tamaño de las advertencias en las cajetillas de cigarros, y un incremento de siete veces en el número de clínicas para dejar de fumar. Como resultado, la comparación de las dos encuestas nacionales de Salud y Nutrición muestra una reducción de 17\% entre 2000 y 2005 en la proporción de adolescentes masculinos que fuma. ${ }^{28}$ Inversiones adicionales en salud pública se han enfocado a mejorar la seguridad humana a través de la vigilancia epidemiológica y una mayor preparación para responder a brotes de enfermedad, desastres y pandemias.

\section{Bienes públicos globales para la toma local de decisiones}

La experiencia de evaluación reunida a través del programa Oportunidades se está aplicando a la reforma actual del sistema de salud. Además de su valor técnico, las evaluaciones rigurosas tienen una utilidad política, ya que ayudan a asegurar la continuidad de las innovaciones en el curso de los cambios de administración. En el caso de Oportunidades, las evidencias científicas persuadieron al gobierno actual no sólo a continuar con el programa, sino a ampliarlo. Los resultados alentadores mostrados por la evaluación continua del Seguro Popular muy probablemente servirán de nuevo para mantener la reforma a través del cambio de gobierno previsto para finales del 2006.

Una marca distintiva de la experiencia mexicana ha sido la inversión sustancial en investigación para diseñar la reforma, monitorear el progreso de su implantación y evaluar sus resultados. Esto es un claro ejemplo de la posibilidad de emplear la ciencia para promover el cambio social mediante la armonización dos valores centrales de la investigación: la excelencia científica y la pertinencia a la toma de decisiones. ${ }^{29}$

El caso mexicano también muestra que el dilema entre la investigación local y mundial es falso (otro puente más que debe tenderse). El proceso de globalización puede convertir el conocimiento en un bien público internacional que puede llevarse al centro de la agenda política nacional para abordar un problema local. ${ }^{30}$ Dicha aplicación, a su vez, realimenta el conjunto global de experiencias, generando así un proceso de aprendizaje compartido entre las naciones. ${ }^{31}$ Asegurar que este ciclo virtuoso realmente se ponga en práctica es una función esencial de la OMS, que lleva a cabo al movilizar la acción colectiva internacional para el bien común de todos los países. ${ }^{32}$

\section{EI $A B C D E$ de una reforma exitosa}

La experiencia mexicana ofrece un caudal de lecciones que se pueden resumir en cinco elementos (cuadro I). Esta experiencia demuestra la posibilidad de tender un puente más: entre análisis y acción. Como ha dicho Donabedian, "...el mundo de las ideas y el mundo de la acción no están separados, como les gustaría que creyéramos algunos, sino que son partes inseparables el uno del otro. Las ideas, en particular, son las verdaderas fuerzas poderosas que le dan forma al mundo tangible". ${ }^{35}$

El camino es claro: las evidencias sólidas deben ser el faro que ilumine el diseño, la implantación y la evaluación de los programas en los gobiernos nacionales, las agencias bilaterales de cooperación y las organizaciones multilaterales. Ésta es la ruta que nos llevará a un desarrollo más equitativo a través de una mejor formulación de las políticas de salud.

\section{Reconocimientos}

El proceso de reforma descrito en este artículo es el resultado del trabajo de un dedicado equipo de la Secretaría de Salud y otras organizaciones en México, que ha contado con el apoyo de diversos socios internacionales. En la preparación de este artículo he recibido análisis y comentarios de Felicia Knaul, Octavio Gómez-Dantés, Jaime Sepúlveda, Rafael Lozano, Eduardo González-Pier, Miguel Ángel Lezana, y Mauricio Hernández-Ávila. Parte de este artículo se basa, con modificaciones, en el discurso inaugural presentado durante el lanzamiento global de la segunda edición de "Disease Control Priorities in Developing Countries", en Beijing el 3 de abril de 2003. 


\section{Cuadro I \\ EL ABCDE dE UNA REFORMA EXITOSA}

Agenda

El primer ingrediente para el éxito es hacer progresar la agenda de la salud en medio de la competencia por la atención y los recursos públicos. Especialmente en su interacción con los ministros de finanzas, las autoridades de salud pueden hacer uso de las evidencias mundiales las cuales muestran que, además de su valor intrínseco, un sistema de salud que funciona bien contribuye al bienestar general de la sociedad al aliviar la pobreza, mejorar la productividad, aumentar las capacidades educativas, desarrollar el capital humano, generar empleo, proteger los ahorros y los activos, aumentar la competitividad y estimular de manera directa el crecimiento económico con una distribución más justa de la riqueza.

Bolsa de recursos

Al colocar a la salud en el centro de la agenda de desarrollo más amplia de una nación, es posible dotarla del grado de prioridad que se merece. Dicha prioridad aumenta el poder de negociación de los ministros de salud en su búsqueda de un mayor apoyo presupuestal. En demasiados países en vías de desarrollo, la inversión actual simplemente no es suficiente para encarar las crecientes demandas impuestas por la doble carga de enfermedad sobre los sistemas de salud. La experiencia mexicana muestra el valor de legislar derechos específicos y los requerimientos de recursos asociados a ellos. Demostrar a través de las evidencias el valor de la salud para el desarrollo puede ayudar a convencer a los tomadores de decisiones a movilizar más dinero para la salud, pero también se debe demostrar la capacidad de generar más salud por el dinero (Ramalingaswami V, comunicación personal).

Capacidad

No existe sustituto para las inversiones de largo plazo en el desarrollo de capacidades. Estos esfuerzos deben enfocarse a dos áreas primordiales. La primera se refiere a la prestación de servicios de salud a través de inversiones en infraestructura física y, más importante aún, en recursos humanos. La segunda tiene que ver con el desarrollo de las instituciones que pueden emprender la investigación necesaria para generar evidencias sólidas para las políticas. En México, la reforma actual ha cosechado los beneficios de 20 años de esfuerzos sostenidos para establecer e impulsar organizaciones como el Instituto
Nacional de Salud Pública y la Fundación Mexicana para la Salud. Estos centros de excelencia han producido investigación pertinente y análisis de políticas, han capacitado investigadores que ocupan cargos claves en la formulación de políticas y han llevado a cabo evaluaciones independientes y creíbles.

Distribución de beneficios

Un ingrediente clave para recabar apoyo público a una reforma es identificar y comunicar sus beneficios específicos. La mejor manera de hacerlo es concentrarse en las enfermedades y los factores de riesgo prioritarios. De esta forma, la gente puede referir conceptos financieros y gerenciales abstractos a beneficios concretos. Una lección fundamental derivada de la experiencia mexicana es que se puede fortalecer la capacidad general del sistema de salud a través de un aumento progresivo de intervenciones preventivas y terapéuticas efectivas contra problemas prioritarios específicos.

Evidencias

Las evidencias derivadas del conocimiento científico confieren poder para transformar los sistemas de salud. Existe un consenso internacional creciente de que la creación y difusión del conocimiento es una de las fuerzas motoras más importantes para el progreso en salud. ${ }^{33,34} \mathrm{Si}$ bien la investigación es un valor en sí mismo, una parte esencial de la cultura, el conocimiento también posee un valor instrumental como medio para mejorar la salud. Esta mejoría se logra a través de tres mecanismos. El primero y más evidente es que el conocimiento se traduce en nuevas y mejores tecnologías, como medicamentos, vacunas y métodos de diagnóstico. En segundo lugar, el conocimiento es internalizado por los individuos, quienes lo utilizan para estructurar sus conductas cotidianas en dominios clave como la higiene personal, los hábitos alimenticios, el comportamiento sexual y la crianza de los hijos. De esta forma, el conocimiento confiere a las personas la facultad para modificar sus estilos de vida y promover su propia salud. El poder derivado del conocimiento también permite que los individuos se conviertan en usuarios informados de los servicios y ciudadanos conscientes de sus derechos. Tercero, el conocimiento se traduce en evidencias que proporcionan los cimientos científicos para la toma de decisiones, tanto en la prestación de servicios de salud como en la formulación de las políticas públicas.

\section{Referencias}

I. United Nations. Millenium Development Goals Report 2005. Nueva York: UN, 2005.

2. Omran AR. The epidemiologic transition: a theory of the epidemiology of population change. Milbank Mem Fund Q 1971;49:509-38.

3. Frenk J, Bobadilla JL, Sepúlveda J, López-Cervantes M. Health transition in middle-income countries: new challenges for health care. Health Pol Plann 1989:4:29-39.

4.World Health Organization. World Health Report 2000. Health systems: improving performance. Ginebra:WHO, 2000.

5. Chen LC, Evans T, Cash R. Health as a global public good. En: Kaul I, Stern M, Grunberg I, eds. Global public goods: international cooperation in the 2 Ist century. Nueva York: Oxford University Press, 1999:284-304. 6. The Mexico statement: strengthening health systems. Lancet 2004;364:1911-1912.
7.World Health Organization. Commission on Macroeconomics and Health. Macroeconomics and health: investing in health for economic development. Ginebra:WHO, 200 I.

8. Frenk J, Sepúlveda J, Gómez-Dantés O, Knaul F. Evidence-based health policy: three generations of reform in Mexico. Lancet 2003;362:1667-I67I. 9. Frenk J, Lozano R, González-Block MA. Economía y salud: propuestas para el avance del sistema de salud en México. Mexico, DF: Fundación Mexicana para la Salud, 1994.

10. Hernández P, Zurita B, Ramírez R, Álvarez F, Cruz C. Las cuentas nacionales de salud. En: Frenk J, ed. Observatorio de la salud. Necesidades, servicios, políticas. México, DF: Fundación Mexicana para la Salud, 1997: 1 19-42.

II. Secretaría de Salud. Hogares con gastos catastróficos por motivos de salud. México 2000. México, DF: Secretaría de Salud, 200I.

12. Knaul FM, Arreola-Ornelas H, Méndez O. Protección financiera en salud: México, 1992 a 2004. Salud Publica Mex 2005;47:430-439.

13. Knaul FM, Arreola-Ornelas H, Méndez O, Miranda M. Preventing impoverishment, promoting equity and protecting households from 
financial crisis: universal health insurance through institutional reform in Mexico. Working Paper. México, DF: Fundación Mexicana para la Salud, 2005 [consultado en: 28 de julio de 2006]. Disponible en: http:// www.gdnet.org/pdf2/gdn_library/awards_medals/2005/ medals_cat3_first.pdf.

14. Xu K, Evans D, Carrin G,Aguilar-Rivera AM. Designing health financing systems to reduce catastrophic health expenditure. Technical briefs for policy-makers no 2. Ginebra:World Health Organization, 2005.

15.Van Doorslaer E, O'Donnell O, Rannan-Eliya RP, Somanathan A, Adhikari SR, Garg CC et al. Effect of payments for health care on poverty estimates in II countries in Asia: an analysis of household survey data. Lancet. 2006;368: I357-I 364.

16. Murray CJL, Frenk J.A WHO framework for health system performance assessment. Bull World Health Org 2000;78:717-31. 17. Knaul FM, Frenk J. Health insurance in Mexico: achieving universal coverage through structural reform. Health Aff 2005;24:1467-76. 18. Secretaría de Salud. Boletín de Información Estadística, núm. 20-25. Mexico, DF: Secretaría de Salud, 2000-2005 [consultado en: 28 de julio de 2006]. Disponible en: http://sinais.salud.gob.mx/sicuentas.

19. Rivera J, Sotres D, Habicht JP, Shamah T, Villalpando S. Impact of the Mexican program for education, health, and nutrition (Progresa) on rates of growth and anemia in infants and young children: a randomized effectiveness study. JAMA 2004;291:2563-570.

20. Gertler P. Do conditional cash transfers improve child health. Evidence from Progresa's control randomized experiment. Am Economic Rev 2004;94:336-4I.

21. Sepúlveda J. Foreword. In: Jamison DT, Breman JG, Measham AR, Alleyne G, Claeson M, Evans DB et al., eds. Disease control priorities in developing countries. 2nd ed. Washington, DC: Oxford University Press for The World Bank, 2006:xiii-xv.

22. Walton DA, Farmer PE, Lambert W, Le' Andre F, Koenig SP, Mukherjee JS. Integrated HIV prevention and care strengthens primary health care: lessons from rural Haiti.J Pub Health Pol 2004;25: 137-58.

23. Instituto Nacional de Estadística, Geografía e Informática, Secretaría de Salud. Bases de datos de mortalidad en México. México, DF: INEGI-
Secretaría de Salud, 2005 [consultado en: 28 de julio de 2006]. Disponible en: http://sinais.salud.gob.mx/mortalidad/mortalidad.htm. 24. Dirección General de Información en Salud. Estadísticas de mortalidad en México: muertes registradas 2003. Salud Publica Mex 2005;47: I7|-87.

25. Secretaría de Salud. Salud: México 200I-2005. Información para la rendición de cuentas. México, DF: Secretaría de Salud, 2006.

26. Baltussen R, Sylla M, Mariotti S. Cost-effectiveness of cataract surgery: a global and regional analysis. Bull World Health Organ 2004:82:338-45

27. Horton R. The coming decade for global action on child health. Lancet 2006;367:3-5.

28. Instituto Nacional de Salud Pública. Encuesta Nacional de Salud y Nutrición 2005-2006. Informe preliminar. Cuernavaca: Instituto Nacional de Salud Pública. Documento de trabajo, 2006.

29. Frenk J, Knaul F, Gómez-Dantés O. Closing the relevance-excellence gap in health research: the use of evidence in Mexican health reform. In: Global Forum update on research for health. Londres: Pro-Brook Publishing, 2004:48-53.

30. Kaul I, Conceiçao P, Le Goulven K, Mendoza R, eds. Providing global public goods: managing globalization. Oxford: Oxford University Press, 2003.

3I. Frenk J, Gómez-Dantés O. Globalization and the challenges to health systems. Health Aff 2002;21:160-65.

32. Jamison DT, Frenk J, Knaul F. International collective action in health: Objectives, functions and rationale. Lancet 1998;351:514-17.

33. World Health Organization. World Health Report 2004. Changing history. Ginebra:WHO, 2004.

34. Jamison D. Investing in health. In: Jamison DT, Breman JG, Measham AR, Alleyne G, Claeson M, Evans DB et al., eds. Disease control priorities in developing countries. 2nd ed.Washington, DC: Oxford University Press for The World Bank, 2006:3-34.

35. Donabedian A. The Baxter American Foundation Prize address. J Health Admin Educ 1986;4:6 II-14. 